ARTICLE HISTORY: Received: September 04, 2021 Accepted: October 28, 2021 Published: November 03, 2021

КОМБИНИРОВАННАЯ МОБИЛЬНАЯ УСТАНОВКА СВЕРХКРИТИЧЕСКОЙ СО2-ЭКСТРАКЦИИ

\author{
В.Гурин ${ }^{1}$, А.Титенко ${ }^{2}$, Д.Старокадомский ${ }^{1,5}$, \\ Куц В. ${ }^{1}$, Л.Демченко ${ }^{3}$, Малочтан С. ${ }^{1}$, Бархоленко В. ${ }^{1}$, \\ М.Решетник, А.Николайчук, Кашуба О. ${ }^{4}$, А.Камарали $^{6}$ \\ 1 - Институт геохимии, минералогии и рудообразования им. М.П. Семененка, НАН Украинь \\ 2- Институт магнетизма НАН і Минобразования Украинь \\ 3 - НТУ «Киевский политехнический институт им.И.Сикорского» \\ 4 - Наииональный Научный Природоведческий Музей НАН, Киев \\ 5 - Институт Химии Поверхности им. акад. А.А.Чуйко, НАН \\ 6 - ГО Центр Общественного Фармаконадзора, Киев
}

\title{
COMBINED MOBILE SUPER CRITICAL CO2 EXTRACTION PLANT
}

\author{
V.Gurin $^{\text {I }}$ A. Titenko ${ }^{2}$, D. Starokadomsky ${ }^{1,5}$, \\ Kuts V. ${ }^{1}$, L.Demchenk ${ }^{3}$, Maloshtan S. ${ }^{1}$, Barkholenko V. ${ }^{1}$, \\ M.Reshetnik ${ }^{4}$, A.Nikolaichuk ${ }^{5}$, Kashuba O. ${ }^{1}$, A.Kamarali $^{6}$ \\ 1 - Institute of Geochemistry, Mineralogy and Ore Formation named after M.P. Semenenka, NAS of Ukraine \\ 2- Institute of Magnetism of the National Academy of Sciences and the Ministry of Education of Ukraine \\ 3 - NTU "I. Sikorsky Kyiv Polytechnic Institute" \\ 4 - National Scientific Natural History Museum of the National Academy of Sciences, Kiev \\ 5 - Institute of Surface Chemistry named after academician A.A. Chuiko, National Academy of Sciences \\ 6 - GO Center for Public Pharmacovigilance, Kiev
}

\begin{abstract}
Аннотация. Представлено детальное описание экспериментальной мобильной установки нового поколения, ее комплектация и возможности сверхкритической $\mathrm{CO}_{2}$-экстракции по переработке растительного сырья. Продемонстрированы новые инженерно-технические решения в работе механической системы, в том числе усовершенствованная конструкция дроссельного вентиля высокого газового давления, вмонтированного в накопители и новации в системе загрузки-выгрузки сырья с использованием съемных стаканов. Указаны рабочие параметры функциональных элементов и их ресурс.

Abstract. A detailed description of an experimental mobile installation of a new generation, its complete set and the possibilities of supercritical CO2-extraction for processing plant raw materials are presented. New engineering solutions in the operation of a mechanical system were demonstrated, including an improved design of a high gas pressure throttle valve installed in storage tanks and innovations in the system for loading and unloading raw materials using removable nozzles. The operating parameters of the functional elements and their resource are indicated.

Ключевые слова: комбинированная мобильная установка, сверхкритическая $\mathrm{CO}_{2}$-экстракция, растительное сырье
\end{abstract}

Keywords: combined mobile unit, supercritical $\mathrm{CO}_{2}$-extraction, plant raw materials

Вступление.

Сверхкритические технологии являются перспективной альтернативой многим существующим технологическим процессам, для функционирования которых требуется создание нового оборудования и соответствующей оснастки, работающей под высоким давлением [1,2]. Развитие сверхкритических флюидных технологий (СКФТ) относится к началу 80-х годов прошлого века и на сегодняшний день характеризуется все более нарастающим интересом. Анализ литературных данных показывает, что за последние двадцать лет технологии, основанные на использовании сверхкритических растворителей, прошли стадию от лабораторных исследований до крупнотоннажных промышленных производств; при этом успешно решаются как технические задачи, так и экологические проблемы современного мира [3]. В свою очередь, сверхкритическая экстракция, интенсивно развиваясь, пришла на замену многим традиционным методам, вытесняя их [1-3]. Технология СКФЭ ориентирована, как правило, на ценные компоненты растительного сырья, содержание которых находиться в небольших количествах (1-3\% от абсолютно сухой массы) и весьма трудно отделяются от природной матрицы. В основу СКФЭ положено свойство газа под воздействием давления и температуры переходить во флюидную фазу, обладающей способностью проницаемости газа и растворимостью присущей жидкости. Свойства сверхкритического $\mathrm{CO}_{2}$ как растворителя можно регулировать - при повышении давления и температуры его растворяющая способность резко увеличивается [4,5]. Для $\mathrm{CO}_{2}$ такими параметрами 
являются (критическая точка $P=7,4 \mathrm{MПа,} T=31,1^{\circ} \mathrm{C}$ ). В техническом аспекте установки СКФЭ значительно удорожаются в связи с технической сложностью проектирования и изготовления необходимых элементов и узлов оборудования. $\mathrm{CO}_{2}$ в состоянии флюида \2\имеет ряд достоинств таких, как: эко-чистота, селективность, отсутствие остаточного растворителя; пожаро- и взрывобезопасность; химинертность (без катализатора); высокая диффузия, низкие вязкость и поверхностное натяжение; общедоступность и низкая стоимость. Важное достоинство - возможность одностадийного получения ценнейших компонентов, которые другими методами не извлекаются - или извлекаются затратно или многостадийно |4-6\. Эти достоинства компенсируются недостаточной мобильностью большинства производимых установок, что требует новых инженернотехнических решений и инвестиций. Широкое применение мобильных экстракционных установок ограничивается техническими проблемами и соответствующей дороговизной. $\mathrm{CO}_{2}$-экстракты получают на экстракционных универсальных модулях, представляющих собой герметические металлические цилиндры, связанные между собой технологическими трубопроводами, снабженными запорной и управляющей арматурой и манометрами.

Новые технические решения и дополнительные возможности легли в основу экстракционного оборудования производимыми мировыми лидерами рынка (таблица 1). Обращает внимание факт концентрации производственных компаний по СКФЭ в Европе, Северной Америке, Азии и на Ближнем Востоке. Рынок в Азии динамично развивается благодаря компаниям-производителям Китая, Южной Кореи и Индии. использованием модульного подхода благодаря, в том числе, громадной сырьевой базе. Если основное внимание в Индии уделяется использованию СКФЭ в производстве пищевых ингредиентов, то Китай имеет приоритет в фитофармацевтических препаратов. Основными производителями $\mathrm{CO}_{2}$ оборудования в России является Ростовский НИЦЭР «Горо», который разработал экспериментальные образцы оборудования [7]. ИВЦ «Инжехим» предлагает системы для осуществления сверхкритической флюидной (СКФ) экстракции собственной разработки (патенты РФ: №157167 и №169873) и производства лабораторного, пилотного и промышленного масштаба (https://ingehim.ru/). География размещения компаний-производителей охватывает все континенты, которые сосредоточены в основном в промышленно развитых странах. Следует отметить тенденцию ввода в эксплуатацию заводов по СКФЭ растительного сырья мощностями переработки 2000 т/год [8] в польском сегменте рынка высоких технологий.

Таблица 1.

Основные мировые производители сверхкритического оборудования.

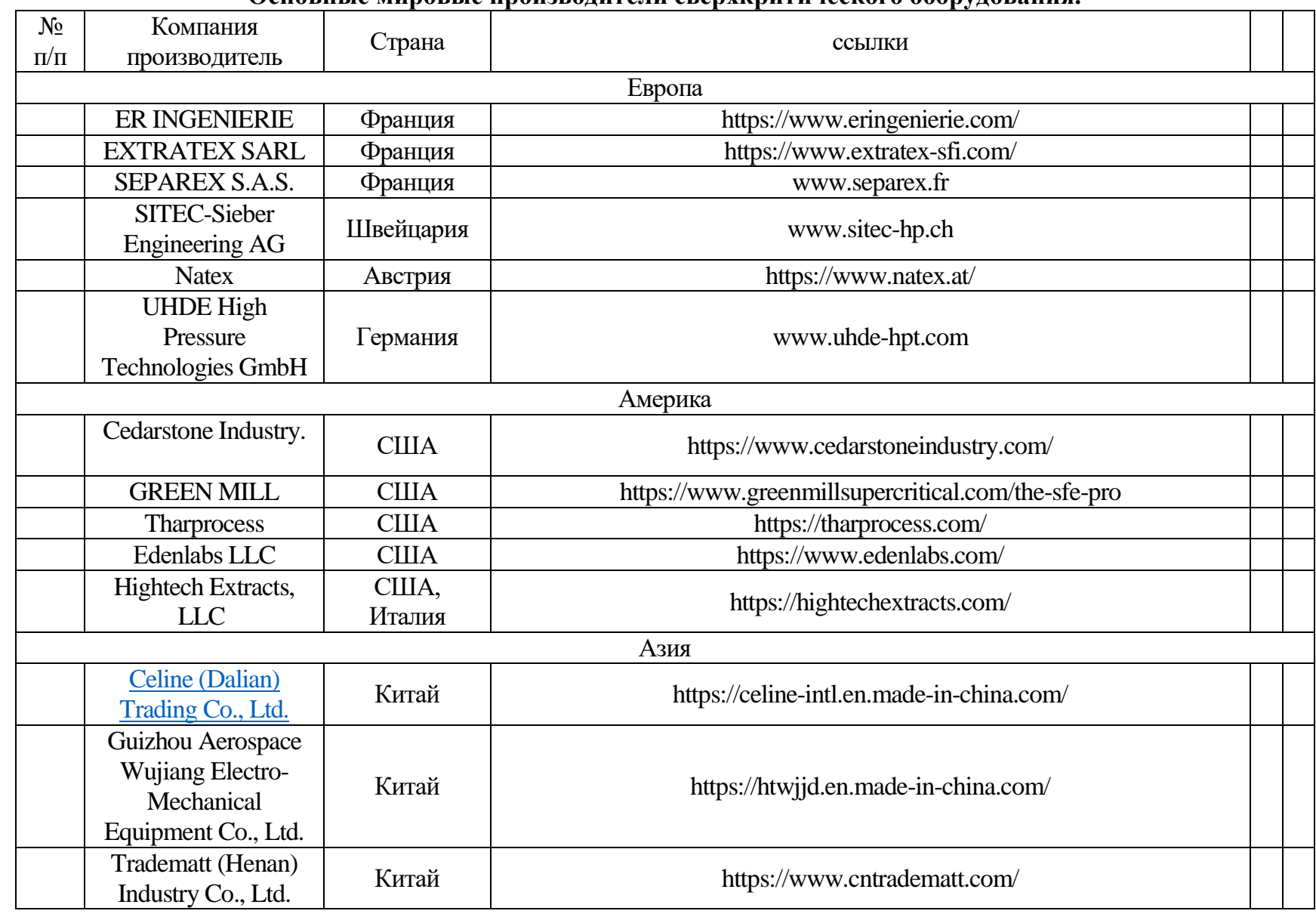




\begin{tabular}{|c|c|c|}
\hline JODA-TECH & Китай & https://www.joda-tech.com/scfe-special/ \\
\hline $\begin{array}{c}\text { BIT HUAN } \\
\text { Supercritical Fluid } \\
\text { Extraction }\end{array}$ & Китай & https://www.hagf.com/ \\
\hline Cedarstone Industry & Китай & https://www.cedarstoneindustry.com/ \\
\hline $\begin{array}{l}\text { Deven Supercriticals } \\
\text { Pvt. Ltd. }\end{array}$ & Индия & https://scfe.en.ecplaza.net/) \\
\hline Pioneer Enterprise. & Индия & https://www.pioneerherbal.com \\
\hline $\begin{array}{c}\text { Supercritical- } \\
\text { Extraction-System }\end{array}$ & Kopeя & $\begin{array}{c}\text { https://www.tradekorea.com/product/detail/P225240/Supercritical- } \\
\text { Extraction-System.html }\end{array}$ \\
\hline НИЦЭР «Горо» & Россия & https://co2-extract-gorofit.ru/ \\
\hline ООО Караван & Россия & http://kuban-co2-karawan.ru/ \\
\hline $\begin{array}{l}\text { ООО «ГОРО- } \\
\text { Инжениринг» }\end{array}$ & Россия & www.extract.ru \\
\hline OOO «TEXAPM» & Украина & (www.texarm.net;) \\
\hline ТОВ КарбЭкс & Украина & scftechnologyl@ukr.net \\
\hline
\end{tabular}

Таблица 2.

Производители и рыночная эффективность сверхкритического оборудования (взято из [12]).

\begin{tabular}{|c|c|c|c|c|}
\hline №№ & $\begin{array}{l}\text { Наименование } \\
\text { производителя }\end{array}$ & Назначение оборудования & $\begin{array}{c}\text { Объем } \\
\text { производства } \\
\text { в год**, US \$ }\end{array}$ & $\begin{array}{l}\text { Ценовые показатели, } \\
\text { US \$ }\end{array}$ \\
\hline 1. & $\begin{array}{l}\text { Thar Technologies, } \\
\text { Inc. (СШA) }\end{array}$ & $\begin{array}{c}\text { Лабораторное и аналитическое оборудование } \\
\text { для работы с СК растворителями }\end{array}$ & 20 млн. & $\begin{array}{c}\text { Лабораторные } \\
\text { установки - от } 75000\end{array}$ \\
\hline 2. & Natex (Австрия) & Промышленное оборудование & 3-5 млн. & \\
\hline 3. & Separex (Франция) & $\begin{array}{c}\text { Оборудование позволяет выполнять весь спектр } \\
\text { исследовательских работ и промышленного } \\
\text { производства }\end{array}$ & & $\begin{array}{c}\text { Лабораторные } \\
\text { установки - от } 200000 \\
\text { Пилотное оборудование } \\
\text { - от } 450000 \\
\text { Промышленное - от } 3,5 \\
\text { млн. }\end{array}$ \\
\hline 4. & SITEC (Швейцария) & $\begin{array}{c}\text { Пилотное оборудование для сверхкритической } \\
\text { экстракции, микронизации, стерилизации. }\end{array}$ & 5-8 млн. & Стоимость от 165000 \\
\hline 5. & $\begin{array}{l}\text { Eden Labs, LLC. } \\
\text { (СШA) }\end{array}$ & Лабораторное оборудование & Нет данных & от 70.000 \\
\hline 6. & Kobelko (Япония) & Промышленное оборудование & Нет данных & \\
\hline 7. & $\begin{array}{l}\text { Chematheur } \\
\text { (Швеция) }\end{array}$ & Промышленное оборудование & Нет данных & \\
\hline 8. & ITT (Индия) & Промышленное оборудование & 2-3 млн. & \\
\hline 9. & Китай & Промышленное оборудование & 15-25 млн. & \\
\hline 10. & Испания & Промышленное оборудование & 2-4 млн. & \\
\hline 11. & $\begin{array}{c}\text { Россия } \\
\text { (предлагаемый } \\
\text { проект) }\end{array}$ & $\begin{array}{c}\text { Лабораторное и пилотное и промышленное } \\
\text { оборудование для СКФ экстракции }\end{array}$ & - & $\begin{array}{c}\text { Лабораторное } \\
\text { оборудование - от } \\
50.000 \\
\text { Пилотное - от } 100.000 \\
\text { Промышленное - от } \\
450.000\end{array}$ \\
\hline
\end{tabular}

Современные устройства СКФ-экстракторов имеют постоянную тенденцию к улучшению и модернизации. Большой спрос также представляет лабораторное, в качестве примера можно привести (HPEP, NOVA--Swiss, Effretikon, Switzerland), но его использование ограничивается только использованием в исследовательских целях.

В отношении мобильных решений установок СКФЭ на данный момент времени известно только одно упоминание об успешно реализованном проекте пилотной мобильной сверхкритической экстракционной 
установки SuperEx. Группа из новой Зеландии [9] установили пилотную экстракционную систему в стандартный морской контейнер, что открывает перспективы для расширения линейки оборудования СКФЭ для работы в нестационарных условиях, в том числе непосредственно в зонах произрастания сырья.

Таким образом, актуальной задачей является разработка мобильного оборудования для глубокой переработки, подразумевающей создание и применение технологий, позволяюших безотходно использовать все компоненты иченного сырья для получения экологичиски чистых продуктов с высокой добавленной стоимостью.

Комбинированная мобильная установка «КарбЭкс»

Наш коллектив имеет опыт научных исследований $[10-11,13]$, и многолетний опыт разработки экстракционного оборудования.

Разработанная нашим коллективом установка $\mathrm{CO}_{2}$-экстракции включает в себя следующие взаимодействующие между собой системы: механическую, обеспечивающую собственно процесс циркуляции $\mathrm{CO}_{2}$ в виде флюида и механизмов управления и контроля в системе, тепловая, обеспечивающая переход $\mathrm{CO}_{2}$ из газообразной в жидкую фазу и наоборот, электронную, обеспечивающую настройки управления и контроля технологическими параметрами процесса экстрагирования, электрическую, обеспечивающую работоспособность компрессора, холодильного оборудования и нагревательных элементов. Правильная компоновка и удобное размещение рабочих элементов экстракционной флюидной СО 2 установки способствуют эффективному экстрагированию исходных продуктов в условиях значительной вариации плотности СО 2 растворителя для экстрагирования различных фракций.

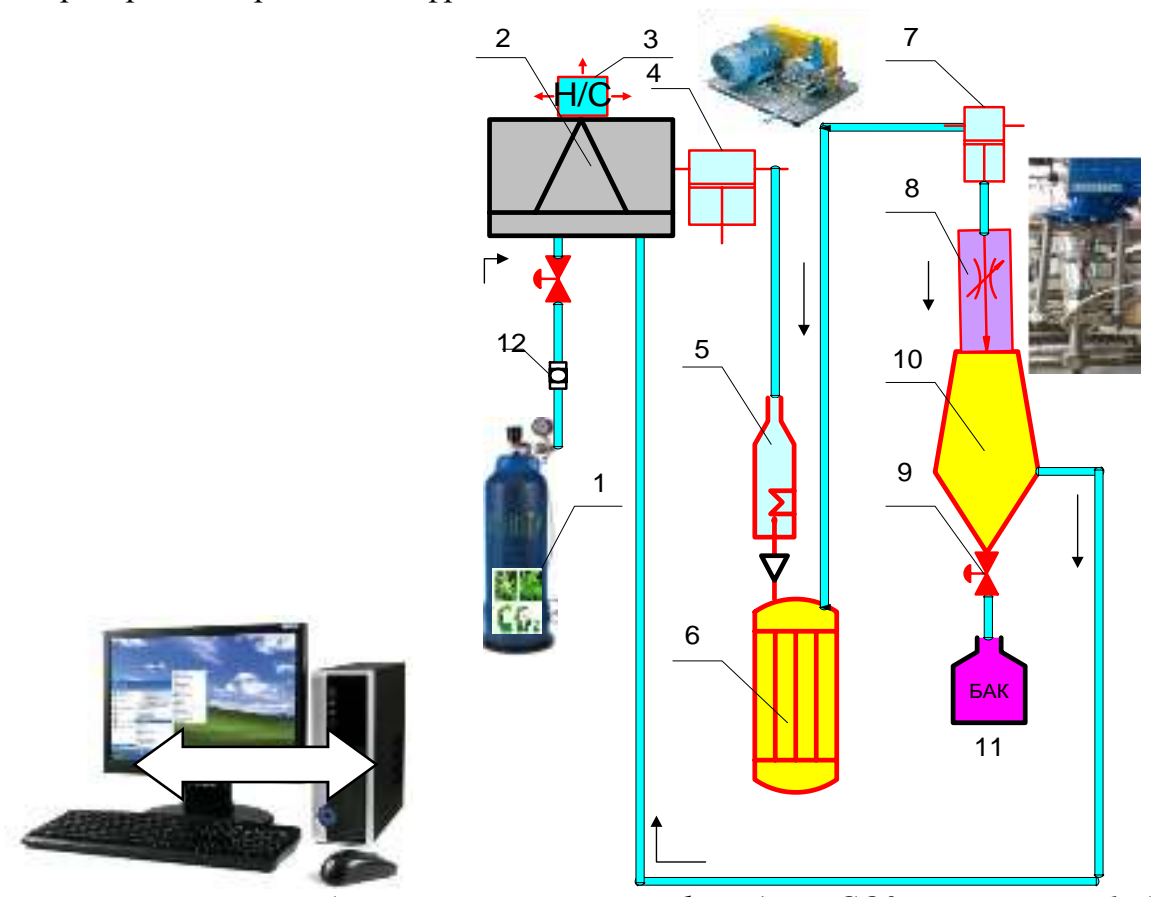

Рис. 1. Принципиальная схема лабораторной установки флюидной СО2 экстракции: 1-баллон СО2;

2-холодильное оборудование; 3- теплообменник; 4 -компрессор ВД; 5-нагреватель; 6- экстрактор;

7- дросселирующий вентиль; 8-изменение потока в дросселирующем вентиле при сбросе ВД; 9 - запорные вентили; 10 - сепаратор; 11 - емкость для принятия экстракта; 12 - фильтр. 


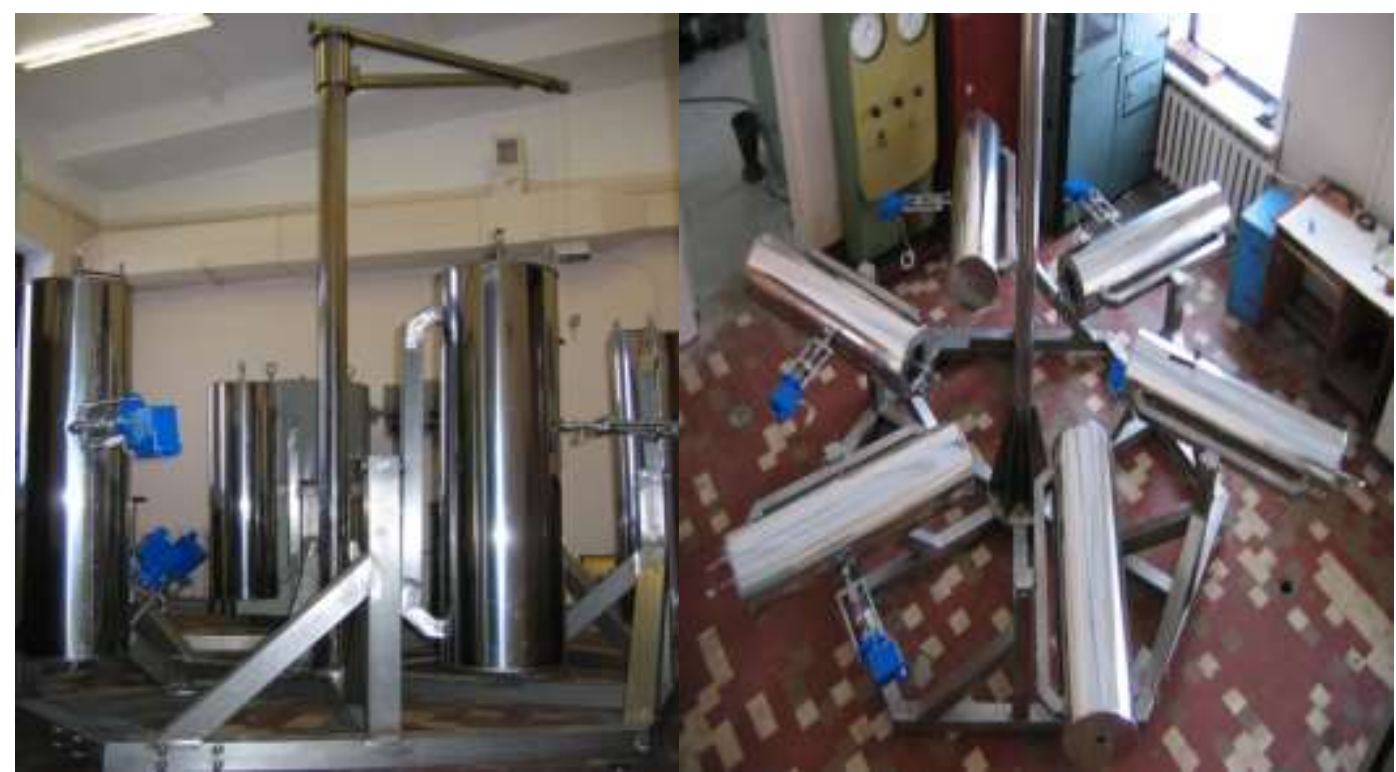

Рис. 2. Промышленная установка СКФ СО2 экстрактов «КарбЭкс 40-і\500» (а - вид с боку; б - вид сверху).

\section{Основные технические характеристики установки следующие.}

1. Экстрактор: 0,5 л, 1 л, 2 л, 5 л/50 МПа, 10 л, 24 л/40 МПа, 40 л/320 МПа, для экстракции как твердых, так и жидких материалов, снабжен рубашкой для циркуляционного нагрева с регулировкой температуры.

2. Сепаратор: 0,3-24 л/30 МПа, 40 л/16-22 МПа, снабжен рубашкой для циркуляционного нагрева с регулировкой температуры.

3. Насос высокого давления: 20/40л МПа, двухплунжерный; 50л/50 МПа, двухплунжерный с частотным регулированием; 400л/40 МПа, трехплунжерный с частотным регулированием;

4. Система охлаждения: снабжена компрессором.

5. Система теплообмена и контроля температуры: в соответствии с технологическими требованиями поставляется с экстрактором и сепаратором. Обеспечивает контроль температуры до $80{ }^{\circ} \mathrm{C}$. Для контроля флюида используется цифровой дисплей. Точность контроля температуры $\pm 0,1^{\circ} \mathrm{C}$.

6. Контроль давления (система безопасности): снабжена электроконтактным датчиком давления на выходе из насоса высокого давления, клапанами сброса давления насоса высокого давления, экстрактора, сепаратора, в соответствии с их максимальным рабочим давлением для автоматического сброса давления в случае его превышения. Экстрактор снабжен системой клапанов обратного давления на выходе, которые контролируют стабильное давление и обеспечивают его точную регулировку. Точность контроля давления в потоке $\pm 0,1$ МПа.

7. Индикатор потока: металлический роторный расходомер с удаленным цифровым дисплеем для контроля мгновенного потока и наработки.

8. Трубопроводы: все емкости, краны, фитинги и трубопроводы, контактирующие с флюидом, изготовлены из нержавеющей стали.

Универсальное оборудование предназначено для выделения полных и фракционных флюидных сверхкритических $\mathrm{CO}_{2}$ экстрактов из твердых и жидких матриц биологического происхождения (корни, цветы, семена, листья и т.п.).

Сферы применения оборудования: фармацевтика, пищевая промышленность, косметическая и химическая отрасль.

В экспериментальную мобильную установку внесены ряд оригинальных технических решений, которые обеспечивают быструю молекулярную очистку экстракционной системы при переходе на другой вид сырья в течение небольшого подготовительного периода;

$>$ Применены гибкие технологические решения: 1 экстрактор - 4 накопителя, 2 экстрактора - 3 накопителя, 3 экстрактора - 2 накопителя, 4 экстрактора - 1 накопитель (переналадка на переработку сырья 10-40 дм³);

$>$ Регулируемое положение экстракторов и экстракторов-накопителей: вертикальное (для твердых матриц), горизонтальное (для жидких матриц);

$>$ Оригинальный подъемный механизм с поворотной стрелой для системы "редуктор-затворстакан"; 
$>$ Оригинальная конструкция редуктора верхнего затвора реактора высокого давления для ускоренной разгрузки-загрузки сырья;

$>$ Автоматизированная система управления и контроля;

$>$ Модульность установки. Возможность подключения дополнительных реакторов высокого давления, блоков сорастворителей, ректификационных колонн;

$>$ Возможность бесперебойной круглосуточной работы в цикле "работа - остановка - работа". Модернизация оборудования за счет переработки сырья в специальных контейнерах. Переоснащение оборудования для получения различных экстрактов при переходе с одной сырья на другую.

$>$ Оригинальное тангенциальное введение экстракционного потока в стакан-циклон накопителя обеспечивает решение различных технологических процессов: микронизация, микрокапсулирования, микрогранулирования, импрегнация, стерилизация, молекулярное очистки изделий различного назначения и др.;

$>$ Оригинальный механизм крепления "стакан - затвор" для оптимизации операций по загрузкеразгрузке сырья и разгрузки продукта;

В конструкцию мобильной установки сверхкритической флюидной $\mathrm{CO}_{2}$ экстракции внесены ряд новых оригинальных технических решений, в частности, конструкция дроссельного вентиля, смонтированного в сборке с накопителем. При помощи механизма, открывающего запорную иглу вентиля, осуществляется подача флюидного экстракта сырья из экстрактора с вариацией давления от $P=40$ МПа (400 атм.) в накопителе до 10 МПа. В результате экстрагирования осуществляется текущий контроль температуры, давления и скорости потока $\mathrm{CO}_{2}$ в экстракторе и накопителе на основании предварительных экспериментов и таким образом подбираются оптимальные размеры проходных сечений вентиля и трубопроводов высокого давления под прогнозируемый технологический цикл процесса эксрагирования исходного сырья. Конструкция вентиля представляет собой достаточно сложную систему перемещения запорной иглы из камеры высокого давления (экстрактор) в камеру низкого давления (накопитель). В конструкцию также входит предохранительный механизм, который имеет возможность блокировки выхода из строя штока и запорной иглы в случае превышения рабочего давления (при превышении рабочих характеристик в системе). На случай изменения объема экстрактора и накопителя при технологических процессах переработки исходного сырья конструкция вентиля изменяется в габаритах и проходных сечениях, исходя из барометрических показателей процесса переработки. Конструкция вентиля позволяет применение запорных игл с различной формой: конусообразных, параболических и т.д. Предложенные технические решения будут детально отражены в соответствующих патентных документах.

Экономически технологии СКФЭ оправданы в случае переработки высокоценного сырья с высоким содержанием БАВ (биологически активных веществ), особенно растений ореолом произрастания, которым является данная территория. В нашем научном арсенале имеется наработки по технологиям переработки: семян льна, чабреца, амаранта, проса, шиповника и отработан способ получения экстрактов из растительного сырья [10].

Процесс экстракции определяется следующими основными показателями:

продолжительность экстракции, температура и давление в экстракторе,

температура и давление в двух сепараторах, скорость потока газовой фазы.

Отработка оптимальных технологических режимов флюидного экстрагирования сырья осуществляется по таким показателям (давление, температура, время цикла, рациональной выход продукта, скорость потока, модуляция потока $\mathrm{CO}_{2}$ и давления, оптимальная влажность и т.п.).

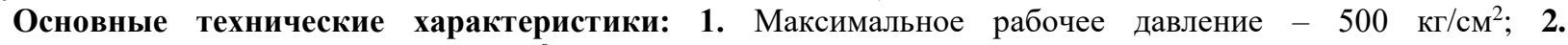
Максимальная рабочая температура $-80^{\circ} \mathrm{C} ; \mathbf{3}$. Максимальное количество фракций $-4 ; \mathbf{4}$. Объем экстрактора 10 дм $^{3} ; \mathbf{5}$. Объем накопителя -10 дм $^{3} ; 6$. Объем экстрактора-накопителя -10 дм³ $^{3}$ 7. Объем фильтра-осушителя 10 дм $^{3} ; \mathbf{8}$. Объем стакана экстракционного -8 дм³ $^{3}$ 9. Объем стакана накопительного -7 дм³ $^{3} ; \mathbf{1 0}$. Объем стакана фильтра-осушителя -8 дм³ ;1. Установленная электрическая мощность - 10 кVA

Отличительные особенности и преимущества оборудования, разработанного в Институте Геохимии $u$ Минералогии HAH 40-i 500 перед однотипным аналогичным оборудованием зарубежного производства:

1. Возможность переналадки оборудования на другой вид сырья в зависимости от требований рынка, в течение небольшого промежутка времени (от 1 до 2 час.), дополнительный монтаж параллельной циркуляционного рукава, обеспечивающего бесперебойную работу системы.

2. Применение оригинальной конструкции автоматизированной системы управления (разработчик ФЛЮЕКС), позволяющей плавно регулировать расход $\mathrm{CO}_{2}$, что способствует изменению плотности флюида и получать экстракты различного состава и качества, а также в зависимости от вида сырья и соответственно программировать процесс управления экстракции. 
3. Технологический процесс СКФЭ с помощью $\mathrm{CO}_{2}$ растворителя требует небольших производственных площадей (от 25 до 50 м²).

4. Малая энергозатратность оборудования от 2 до 15 кВт.

Таким образом, разработанная установка СКФ-экстракции в полной мере соответствует основным критериям экологичности, экономичности, безопасности для работ по переработке растительного сырья по стандартам, предъявляемых к натуральным пищевым ингредиентам в ее мобильном исполнении. Представленная установка расширяет данный класс оборудования и позволяет экстрагировать многочисленные виды растительного сырья и конкурировать с известными аналогами.

В нашем арсенале имеются возможности производства СКФ оборудования и оптимизация технологического цикла экстрагирования различных видов режимов СКФ-технологии на качественный и количественный состав экстрактов, обозначены подходы к регулированию параметров технологии с целью оптимизации процесса для наиболее полного извлечения БАВ из растительного сырья.

\section{Выводы:}

1. Производителями оборудования СКФ $\mathrm{CO}_{2}$ экстракции являются высокотехнически развитые страны с огромным промышленным потенциалом и инженерно-конструкторской инфраструктурой, которые имеют свои компании с замкнутым производственным циклом.

2. Предложенная модель установки $\mathrm{CO}_{2}$ экстракции имеет ряд преимуществ над известными аналогами и создает конкурентные возможности для перспективного использования в качестве альтернативного мобильного оборудования, как на внутреннем, так и внешних рынках.

\section{Цитируемая литература}

1. Brunner, G. (1994). Gas extraction: an introduction to fundamentals of supercritical fluids and the application to separation processes. New York: Springer. http://dx.doi.org/10.1007/978-3-662-07380-3.

2. M.A. McHugh, V.J. Krukonis, Supercritical Fluid Extraction: Principles and Practice, Butterworths, Boston, 1994.

3. M.M.R. de Melo, A.J.D. Silvestre, C.M. Silva, Supercritical fluid extraction of vegetable matrices: Applications, trends and future perspectives of a convincing green technology, J. Supercrit. Fluid. 92 (2014) 115-176. http://dx.doi.org/10.1016/j.supflu.2014.04.007.

4. Michael P. Ekart, Karen L. Bennett, Susan M. Ekart, Gurdev S. Gurdial, Charles L. Liotta, Charles A. Eckert Cosolvent interactions in supercritical fluid solutions, v.39, p. 235-248, 1993. https://doi.org/10.1002/aic.690390206

5. Mukhopahyay M. Natural Extracts Using Supercritical Carbon Dioxide. CRC Press LLC, New York, $2000,339 \mathrm{p}$.

6. C.A. Ecker, B.L. Knutson. Fluid Phase Equilib., v. 83, p. 93>100, 1993.

7. Горофиты. Интернет-магазин СК-СО2 экстрактов для производства косметических средств. https://co2-extract-gorofit.ru/ .Инженерно-Внедренческий Центр «Инжехим» (ИВЦ «Инжехим») https://ingehim.ru/

8. Supercritical $\mathrm{CO}_{2}$ extraction and its applications Edited bydr hab. inż. Edward Rój, prof. nadzw. Lublin 2014 Chapter January 2014 DOI: 10.13140/2.1.3565.9207

9. Owen Catchpole; Stephen Tallon ANNUAL REPORT. http://www.irl.cri.nz

10. СПОСІБ ЕКСТРАКЦІЇ РОСЛИННОЇ СИРОВИНИ Патент на корисну модель 129354 25.10.2018, бюл. № 20

11. D.Starokadomsky et al.”New Combined Mobile Supercritical CO2-extraction Unit with Innovation Technological Elements" $\backslash \backslash$ GE\&RA International Research Conference 2021 to be held on October 01 and 2, 2021 . Technical Session-1 "Leading the change: Embracing New Trends and Innovations in Health Sciences, Education, Nursing and Technology", https://www.youtube.com/watch?v=PNW34S2dnNw (at 2h30-2h35min)

12. Инновации бизнесу. Краткое описание заменяемого процесса или решаемой проблемы. http://www.ideasandmoney.ru/Ppt/Details/297418

13. ОБЗОР НАУЧНЫХ РАБОТ ПО ТЕХНОЛОГИЯМ ЭКСТРАГИРОВАНИЯ БИОКОМПОНЕНТОВ ИЗ РАСТИТЕЛЬНОГО СЫРЬЯ. СВЕРХКРИТИЧЕСКАЯ СО2-ЭКСТРАКЦИЯ - ЭФФЕКТИВНЫЙ НОВЫЙ МЕТОД РЕШЕНИЯ ГЛОБАЛЬНОЙ ПРОБЛЕМЫ УТИЛИЗАЦИИ И КАЧЕСТВА РАСТИТЕЛЬНОГО И ОРГАНИЧЕСКОГО СЫРЬЯ. ॥ Д.Старокадомский, А.Титенко, А.Камарали, Куц В., Малоштан С,Бархоленко В., Кашуба О., М.Решетник, Старокадомская А., Диамант В., Шульга С., В.Гурин \\ GLOBUS: Технические науки. T..7, № 3(39). - c.25-35. - DOI: 10.52013/2713-3079. 


\section{References}

1. Brunner, G. (1994). Gas extraction: an introduction to fundamentals of supercritical fluids and the application to separation processes. New York: Springer. http://dx.doi.org/10.1007/978-3-662-07380-3.

2. M.A. McHugh, V.J. Krukonis, Supercritical Fluid Extraction: Principles and Practice, Butterworths, Boston, 1994.

3. M.M.R. de Melo, A.J.D. Silvestre, C.M. Silva, Supercritical fluid extraction of vegetable matrices: Applications, trends and future perspectives of a convincing green technology, J. Supercrit. Fluid. 92 (2014) 115-176. http://dx.doi.org/10.1016/j.supflu.2014.04.007.

4. Michael P. Ekart, Karen L. Bennett, Susan M. Ekart, Gurdev S. Gurdial, Charles L. Liotta, Charles A. Eckert Cosolvent interactions in supercritical fluid solutions, v.39, p. 235-248, 1993. https://doi.org/10.1002/aic.690390206

5. Mukhopahyay M. Natural Extracts Using Supercritical Carbon Dioxide. CRC Press LLC, New York, 2000, 339 p.

6. C.A. Ecker, B.L. Knutson. Fluid Phase Equilib., v. 83, p. 93>100, 1993.

7. Gorofity. Internet-magazin SK-SO2 ekstraktov dlya proizvodstva kosmeticheskikh sredstv. https://co2-extract-gorofit.ru/ .Inzhenerno-Vnedrencheskiy Tsentr «Inzhekhim» (IVTS «Inzhekhim») https://ingehim.ru/

8. Supercritical $\mathrm{CO}_{2}$ extraction and its applications Edited bydr hab. inż. Edward Rój, prof. nadzw. Lublin 2014 Chapter January 2014 DOI: 10.13140/2.1.3565.9207

9. Owen Catchpole; Stephen Tallon ANNUAL REPORT. http://www.irl.cri.nz

10. SPOSIB EKSTRAKTSIYI ROSLYNNOYI SYROVYNY Patent na korysnu model' 129354 25.10.2018, byul. № 20

11. D.Starokadomsky et al.”New Combined Mobile Supercritical CO2-extraction Unit with Innovation Technological Elements" $\backslash \backslash$ GE\&RA International Research Conference 2021 to be held on October 01 and 2, 2021 . Technical Session-1 "Leading the change: Embracing New Trends and Innovations in Health Sciences, Education, Nursing and Technology", https://www.youtube.com/watch?v=PNW34S2dnNw (at 2h30-2h35min)

12. Innovatsii biznesu. Kratkoye opisaniye zamenyayemogo protsessa ili reshayemoy problemy.http://www.ideasandmoney.ru/Ppt/Details/297418

13. REVIEW OF SCIENTIFIC WORKS ON TECHNOLOGIES FOR EXTRACTION OF BIOCOMPONENTS FROM VEGETABLE RAW MATERIALS. SUPER CRITICAL CO2 EXTRACTION IS AN EFFECTIVE NEW METHOD FOR SOLVING THE GLOBAL PROBLEM OF UTILIZATION AND QUALITY OF PLANT AND ORGANIC RAW MATERIALS. D. Starokadomsky, A. Titenko, A. Kamarali, Kuts V., Maloshtan S, Barkholenko V., Kashuba O., M. Reshetnik, Starokadomskaya A., Diamant V., Shulga S., V. Gurin II GLOBUS: Technical Science. Vol..7, № 3(39). - pp.25-35. - DOI: 10.52013/2713-3079. 